\title{
Future Directions of Research in Geometry: A Summary of the Panel Discussion at the 2007 Midwest Geometry Conference ${ }^{\star}$
}

Edited by Lawrence J. PETERSON

University of North Dakota, Grand Forks, North Dakota, USA

E-mail: lawrence_peterson@und.nodak.edu

URL: http://www. und.nodak.edu/instruct/lapeters

Received August 09, 2007; Published online August 15, 2007

Original article is available at http://www.emis.de/journals/SIGMA/2007/081/

\begin{abstract}
The 2007 Midwest Geometry Conference included a panel discussion devoted to open problems and the general direction of future research in fields related to the main themes of the conference. This paper summarizes the comments made during the panel discussion.

Key words: determinants; differential complexes; differential geometry; Einstein metrics; GJMS operators; global invariants; heat kernel; Kähler metrics; $Q$-curvature; Sobolev inequalities
\end{abstract}

2000 Mathematics Subject Classification: 53A35

Dedicated to the memory of Thomas P. Branson

The 2007 Midwest Geometry Conference was held at the University of Iowa in Iowa City, Iowa, USA (May 18-20, 2007). In addition to the usual talks given at such meetings, the conference also included a panel discussion devoted to open problems and the general direction of future research in geometry and related fields. This paper is an edited summary of the panel discussion and the contributions of the members of the audience.

The panel included Sun-Yung Alice Chang (Princeton), Michael Eastwood (Adelaide), A. Rod Gover (Auckland), C. Robin Graham (University of Washington), Rafe Mazzeo (Stanford), Bent Ørsted (Aarhus), and Paul Yang (Princeton). The audience members who spoke were James G. Branson (University of California, San Diego), Stephen Fulling (Texas A \& M), Colin Guillarmou (Nice), Claude LeBrun (Stony Brook), Andrew Waldron (University of California, Davis), and Shihshu Walter Wei (Oklahoma). Rafe Mazzeo chaired the panel.

Most of the panel discussion related to the main theme of the 2007 Midwest Geometry Conference, namely the work of Thomas P. Branson (1953-2006). The panel and the audience discussed individual open problems and topics that people will likely investigate in the years to come. An edited summary of this discussion follows. We provide the various comments in approximately the same order that they occurred during the actual group discussion. The discussion began with some remarks by Rafe Mazzeo.

Rafe Mazzeo: Three prominent topics have come up in the course of the 2007 Midwest Geometry Conference so far, namely

- Branson's Q-curvature;

- Poincaré-Einstein metrics;

- Bach-flat metrics.

${ }^{\star}$ This paper is a contribution to the Proceedings of the 2007 Midwest Geometry Conference in honor of Thomas P. Branson. The full collection is available at http://www.emis.de/journals/SIGMA/MGC2007.html 
James Branson: What does all of this have to do with physics?

Editor's note: For a partial answer to this question, see the replies by Andrew Waldron and Stanley Deser near the end of this paper. After the question by Jim Branson, the discussion turned to some specific open problems.

Claude LeBrun: Are there compact four-dimensional manifolds which are Bach-flat and neither

1) locally conformally Einstein nor

2) half flat, i.e. self-dual or anti-self-dual?

It might be reasonable to start by investigating the toric case, that is, by considering only those metrics which admit an isometric $U(1) \times U(1)$ action.

Another open problem is to classify asymptotically locally Euclidean scalar-flat Kähler metrics on complex surfaces. Spaces of this type turn out to naturally arise as blow-up limits in many problems related to the convergence of sequences of Bach-flat metrics, and a complete classification would provide a powerful tool for proving that curvature can only accumulate and bubble off in very specific circumstances.

Alice Chang: A very general question is to ask "What is the geometric content of $Q$ curvature?" For example, we know that one can associate the scalar curvature with the conformally invariant constant called the "Yamabe constant". When this constant is positive, it describes the best constant (in a conformally invariant sense) of the Sobolev embedding of $W^{1,2}$ into $L^{2 n /(n-2)}$ space; this in itself can be viewed as a $W^{1,2}$ version of the isoperimetric inequality. It would be interesting to know if $Q$-curvature, or the conformally invariant quantity $\int Q$ associated with it, satisfies some similar inequalities with geometric content.

Mike Eastwood: It would be good to have a characterization of $Q$-curvature. Although we know that $Q$-curvature has many properties, we do not understand enough of its properties to characterize it.

Alice Chang: It would be nice to have a uniqueness result which would characterize $Q$ curvature.

Mike Eastwood: One can use the Fefferman-Graham ambient metric to canonically construct Branson's $Q$-curvature. Why is this so?

Alice Chang: Can one describe the "renormalized volume" in terms of the ambient metric?

Robin Graham: Concerning Mike Eastwood's request for a characterization of $Q$-curvature, I made a conjecture of such a characterization at the workshop on "Conformal structure in geometry, analysis, and physics" at the American Institute of Mathematics in 2003. See problems 10 and 11 of the open problems list available at

http://www. aimath.org/pastworkshops/confstruct.html

To my knowledge, this conjectured characterization is still open and, if true, would provide a good characterization of $Q$-curvature. The conjecture comes in two parts. The first part is a conjectured characterization of the critical "GJMS" operator, which appears in the conformal transformation law for $Q$-curvature. The GJMS construction shows that the coefficients of each GJMS operator can be written just in terms of the Ricci tensor and its covariant derivatives, and that the $Q$-curvature can likewise be written just in terms of the Ricci tensor and its covariant derivatives. My specific two-part conjecture is as follows.

1. Let $n \geq 4$ be even. Suppose that $P$ is a scalar natural operator in dimension $n$ whose principal part is $\Delta^{n / 2}$. Suppose also that $P$ satisfies the same conformal covariance relation as the critical GJMS operator. If all of the coefficients of $P$ can be written in terms of the Ricci tensor and its covariant derivatives, then $P$ is the critical GJMS operator.

2. A local scalar conformal invariant of weight $-n$ which can be written only in terms of the Ricci tensor and its covariant derivatives must vanish. 
If true, the first conjecture would uniquely characterize the critical GJMS operator, and then the second would imply that $Q$-curvature is uniquely characterized by its conformal transformation law involving this critical GJMS operator. Both conjectures are true in dimensions 4 and 6 .

Notes: The GJMS operators are the conformally covariant partial differential operators of Graham, Jenne, Mason, and Sparling [6]. The critical GJMS operator is the operator of order $n$ in even dimension $n$.

Claude LeBrun: Another open problem is to characterize those Kähler metrics for which the Fefferman-Graham obstruction tensor vanishes. The goal would be to do this for arbitrary dimensions. In real dimension four, the Fefferman-Graham obstruction tensor is just the Bach tensor, and in [4], Andrzej Derdzinski showed that it vanishes for a Kähler metric if and only if the metric is conformally Einstein on the open set where the scalar curvature is nonzero. This observation plays a central role in my joint work with Chen and Weber [2], as I described in my lecture yesterday. Since the Fefferman-Graham obstruction tensor exactly represents the gradient of the total $Q$-curvature, and since many differential geometric problems simplify when restricted to the Kähler arena, this might be an excellent context in which to obtain a more concrete understanding of these higher-order curvature objects, which we now but glimpse through a glass, darkly.

Rod Gover: An emerging theme in my more recent work with Tom Branson was the construction of a new class of differential complexes that we termed "detour complexes". These complexes exist on, for example, even-dimensional conformally flat manifolds and are conformally invariant. They are related to (but distinct from) Bernstein-Gelfand-Gelfand (BGG) complexes. In Riemannian signature, the cases treated yield elliptic complexes. One open problem is to show that all conformal detour complexes are elliptic. So far there are constructions of several families of curved analogues. The general theory of curved analogues should be developed.

Related to the de Rham detour complexes is a generalization of $Q$-curvature. In my joint work with Tom, we obtained what are termed " $Q$-operators". These exist on even-dimensional conformal manifolds; they act on closed forms and determine conformally invariant cohomology pairings. Any progress in understanding $Q$-operators would be extremely interesting. In the case of zero-forms, the $Q$-operators recover the usual $Q$-curvature, and the cohomology pairing in that case is just the integral of the $Q$-curvature. On conformally flat spaces this recovers the Euler characteristic. So an important question is whether on conformally flat structures the $Q$-operators have a link to topology which generalizes this.

The $Q$-operators yield problems which generalize the problem of prescribing constant $Q$ curvature. In these problems, one begins with a conformal class of metrics. One then seeks a metric in this conformal class and a closed differential $k$-form which, for this particular metric, is an eigenform for the $Q$-operator on $k$-forms. The eigenvalue is the analogue of constant $Q$. It would be interesting to know if this yields a manageable PDE problem.

Bent Ørsted: In the years to come there will be further research in Sobolev inequalities. People will study strong Hardy-Littlewood-Sobolev inequalities and their analogues in the context of Cauchy-Riemann (CR) geometry and other parabolic geometries. One goal will be to maximize or minimize linear functionals.

Tom Branson was interested in calculating determinants of matrices in the setting of intertwining operators; when the $K$-types have higher multiplicity, an intertwining operator is not just a number for each $K$-type, but rather a matrix, and one would like to compute its determinant. This has been studied by Wallach, Cohn, and Vogan. See [11], for example. There is some hope that the method of spectrum generating could give results in this direction. Actually, Branson had some computations for a multiplicity-two example involving differential forms.

Paul Yang: It would be interesting to study $Q$-curvature in odd dimensions. The plan would be to define the GJMS operator as a nonlocal operator in the context of Poincaré- 
Einstein metrics as defined by Fefferman and Graham. This should provide interesting analytic questions.

Robin Graham: Spyros Alexakis reports that he has characterized scalar Riemannian invariants whose integral over a compact manifold is a conformal invariant. He has characterized them as a linear combination of a divergence, a multiple of the Pfaffian, and a local conformal invariant of weight $-n$, where $n$ is the dimension. It is an interesting question whether such a decomposition is unique. It is always unique in dimensions 4 and 6 .

Rod Gover: For suitable conformally invariant differential operators and for operators or complexes with appropriate "nearly invariant" properties, there are determinants (or torsions) which are not conformally invariant but nevertheless have useful "Polyakov type" formulas for their conformal variation. In my joint work with Tom Branson, Tom has proposed using invariant theory and related tools to investigate the existence of a universal form for such formulas.

Robin Graham: Neil Seshadri has defined a torsion in the contact (CR) case analogous to the conformal half-torsion. See [9]. Seshadri's torsion also has a local variation under change of contact form and almost complex structure, which is given in terms of heat kernel coefficients. Further work needs to be done to make this more explicit and to better understand such formulas.

Bent Ørsted: In addition to the above problems, people should study other invariants as well.

Stephen Fulling: Ten to twenty years ago, many people, including Tom Branson, devoted a great deal of attention to calculating heat kernel coefficients explicitly to the highest order feasible. About ten years ago we all seemed to get tired of it, but it may now be time to look at the subject again. It seemed to me that we were right on the verge of reaching an understanding of the structure at some more profound algebraic or combinatorial level.

In the case of a Schrödinger operator with just a potential (in flat space) one can write down an explicit combinatorial formula for the $n$ 'th coefficient, even before passing to the diagonal and taking the trace. For a Riemannian manifold, a computer can in principle grind out the formulas recursively, but the result is not illuminating. The problem arises in reducing the formula to a canonical form. There is an accumulation of terms of high degree in the Riemann tensor coming from the commutation of covariant derivatives in the process of making the lowdegree terms linearly independent; at the end, the coefficient of such a term (a sum of many disparate contributions) conveys no clear information. Yet there could still be something to learn here.

Shihshu Walter Wei: My question is related to Professor Alice Chang's question: Is there any connection between $Q$-curvature and $p$-harmonic geometry? For example, let us recall that a manifold $M$ is $p$-hyperbolic, $1 \leq p<\infty$, if the $p$-capacity of any compact subset is positive (or equivalently, if $M$ supports a nonconstant positive $p$-superharmonic function) and $p$-parabolic otherwise. If $p=n=\operatorname{dim} M$, then $n$-parabolicity of $M$ is a (quasi)conformal invariant (cf. [10]). We know that if the sectional curvature of a complete simply-connected manifold $M$ is bounded above by a negative constant, then $M$ supports an isoperimetric inequality, and via isoperimetric profile we see that the manifold is $p$-hyperbolic for every $p \geq 1$ (cf. e.g. [1]). On the other hand, we also know that the Brunn-Minkowski inequality in the theory of convex bodies is connected with other fundamental inequalities, such as the isoperimetric, Sobolev, and Prékopa-Leindler inequalities, and can be extended to the $p$-capacity of convex bodies, $1<p<n$ (cf. [3]).

It would be interesting to know if $Q$-curvature, or the conformally invariant quantity $\int_{M} Q$ associated with it, is linked to $p$-parabolicity, $p$-hyperbolicity, $p$-capacity, $p$-energy, or other functionals arising in the context of calculus of variations, the theory of PDEs, or geometric measure theory, or satisfies some inequality of geometric significance.

Colin Guillarmou: Another interesting problem is to study the dimension of the kernel of the critical GJMS or Branson-Gover operators. The dimension of the kernel of the GJMS operators is a conformal invariant. It appears in the Selberg trace formula for (non-compact) 
convex co-compact hyperbolic manifolds. These manifolds have (locally conformally flat) conformal infinity. Thus the natural GJMS operators and the dimension of their kernels show up in the trace formula (see [7] and [8]). Note also that the kernel of the critical GJMS operator is a conformally invariant space. The same problem for the Branson-Gover operators on forms is also a parallel thing to consider.

It is also of interest to study the determinant of the GJMS operators. Branson, Chang and Yang worked on the Yamabe and square of Dirac cases. It could be worthwhile to look at higherorder GJMS operators and see if it is possible to obtain results in the same spirit as in the case of the determinant of the Laplacian. For instance, it is possible to compute the determinant of the GJMS operators explicitly for a class of odd-dimensional locally conformally flat manifolds. (See [7].)

Robin Graham: Over the last few years, there have been a number of constructions of global invariants of contact manifolds which arise (or are expected to arise) as integrals of local invariants of a choice of contact form and compatible almost complex structure. For example, Hirachi, Boutet de Monvel, Ponge, Seshadri, and Biquard-Herzlich-Rumin have developed constructions of such global invariants. None of these global invariants is known to be nonzero, and some of them have been shown to vanish.

This gives rise to a natural conjecture about such invariants, which is formulated explicitly by Seshadri in Appendix A of [9]. The conjecture is motivated by a result of Gilkey which goes as follows. Consider any local scalar Riemannian invariant whose integral over a compact manifold is independent of the metric. This invariant is necessarily a divergence plus a multiple of the Pfaffian; the integral must therefore be a multiple of the Euler characteristic.

The analogous conjecture in the contact case reads as follows. Suppose that one has a local scalar invariant of a contact manifold together with a choice of contact form and compatible almost complex structure whose integral over a compact manifold is a contact invariant. Then the local invariant is a divergence, and hence the integral must vanish.

A positive answer to this conjecture would indicate a unified approach to showing that all the invariants which arise in the above-mentioned constructions necessarily vanish. It would also show that there is no contact analogue of the Pfaffian.

Claude LeBrun: It seems worth pointing out that the results Robin just alluded to only hold if one requires the global invariant to be independent of the orientation of the manifold; in particular, the hypotheses exclude things like Pontrjagin numbers. Perhaps one should try to classify invariants for which the invariant is allowed to change sign when the orientation of the manifold is reversed. Next, one might ask what happens when the dependence on orientation is allowed to be arbitrary.

Andrew Waldron: It would be interesting to study Lorentzian versions of many of these questions. Our universe has Lorentzian signature. Many formulas look exactly the same in Lorentzian and Riemannian signatures; other aspects differ significantly, however, especially when one wants to solve, rather than just write down, interesting systems of PDEs on curved manifolds.

Rod Gover: It would be interesting to consider the above questions in the context of special structures such as almost Einstein metrics.

Editor's note: As we noted above, Jim Branson posed the question "What does all of this have to do with physics?" In the weeks following the 2007 Midwest Geometry Conference, Stanley Deser, of Brandeis University, has provided us with a short essay which comments on ways in which Tom Branson's work relates to physics. Andrew Waldron has also provided additional comments. Waldron's comments and Deser's essay follow.

Andrew Waldron: There is a deep relationship between conformal geometry on the one hand and the AdS/CFT correspondence in physics on the other. The latter, first suggested by Juan Maldacena, relates gravity/string theories in an $(n+1)$-dimensional asymptotically 
Anti de Sitter background to conformal field theories on their boundaries. In particular the $Q$-curvature and Poincaré metric are related to conformal anomalies of the boundary conformal field theories.

Note: AdS/CFT means "Anti de Sitter/conformal field theory".

Stanley Deser's Essay: Tom Branson - An Appreciation from Physics.

Theoretical physicists are supposed to know, at least vaguely, some of the mathematical riches available to help our research. The reality is often rather different. First you try to locate someone in that distant country who can help you, if only you can figure out how to frame your vague queries intelligibly in his or her language. And then, if you are lucky, you just have to translate back the usually far too technical answer.

Tom Branson was the exception I was lucky enough to encounter early enough in a project on conformal anomalies, a subject of great physical interest. True, he did at first refer me to his papers or quote theorems - all interaction was by email or (rarely) by phone - but he wisely realized that I needed words of one syllable explanations, and he cheerfully provided them.

I will be monosyllabic myself in describing the context; a paper that provides technical and citation details is [5]. Conformal anomalies are described by effective actions in (even) dimension $D$ that hinge on very special operators such as the inverse Laplacian in $D=2$ and the Paneitz operator - the self-adjoint conformal completion of the inverse square Laplacian in $D=4$. Both Tom and Paneitz (who also died tragically, already as a graduate student) were students of Irving Segal at MIT, where Irving's passion for all things conformal inspired them. Actually, there are two different anomaly types with very different properties and origins. The Paneitz operator and its generalizations (to which Tom also contributed) arise in type A. Type $\mathrm{B}$ requires a very different approach, and only starts at $D=4$. Here the desired operators are not only bilocal but heavily tensorial, unlike the scalar ones of type A.

This is where Tom really saved the day. It was morally certain to a physicist that there had to exist a fourth derivative order (in $D=4$ ) of tensor rank eight which when acting to any power on a tensor with the symmetries of the conformal curvature (Weyl) left it conformally invariant. It was Tom who actually had the desired object (and of course its generalizations for arbitrary $D$ ) and who told me that he and Rod Gover were going to publish it. Talk of mining mathematical treasure with no effort! This work enabled me to to give the closed form solution to the type B effective action, whose original discovery in 1976 was only given as a hand-waving scalar approximation to the real thing.

Emboldened by these riches, I was pumping Tom for many other important unfilled physics needs that would have propelled us, when the terrible news of his death came. Despite the action-at-a-distance nature of our relation, the loss was felt as one of an old friend!

\section{Acknowledgements}

This paper is based in part on notes the editor took during the panel discussion at the 2007 Midwest Geometry Conference. The editor would like to thank Alice Chang, Stanley Deser, Mike Eastwood, Stephen Fulling, Rod Gover, Robin Graham, Colin Guillarmou, Palle Jorgensen, Claude LeBrun, Rafe Mazzeo, Bent Ørsted, Andrew Waldron, Shihshu Walter Wei, and Paul Yang for the additional notes and clarification that they provided in the weeks following the conference and panel discussion.

This material is based upon work supported by the National Science Foundation under grants No. DMS-0509068 and No. PHY04-01667.

Any opinions, findings, and conclusions or recommendations expressed in this material are those of the authors and do not necessarily reflect the views of the National Science Foundation. 


\section{References}

[1] Chen B.Y., Wei S.W., Geometry of submanifolds of warped product Riemannian manifolds $I \times{ }_{f} S^{m-1}(k)$ and applications to $p$-harmonic and quasiregular mappings, submitted.

[2] Chen X., LeBrun C., Weber B., On conformally Kähler, Einstein manifolds, arXiv:0705.0710.

[3] Colesanti A., Salani P., The Brunn-Minkowski inequality for p-capacity of convex bodies, Math. Ann. 327 (2003), 459-479.

[4] Derdziński A., Self-dual Kähler manifolds and Einstein manifolds of dimension four, Comp. Math. 49 (1983), 405-433.

[5] Deser S., Conformal anomalies revisited: closed form effective actions in $D \geq 4$, Nuclear Phys. B Proc. Suppl. 88 (2000), 204-209.

[6] Graham C.R., Jenne R., Mason L.J., Sparling G.A.J., Conformally invariant powers of the Laplacian, I: existence, J. London Math. Soc. (2) 46 (1992), 557-565.

[7] Guillarmou C., Generalized Krein formula and determinants for even dimensional Poincaré-Einstein manifolds, math.SP/0512173.

[8] Patterson S.J., Perry P.A., The divisor of Selberg's zeta function for Kleinian groups, Appendix A by Charles Epstein, Duke Math. J. 106 (2001), 321-390.

[9] Seshadri N., Some notes on analytic torsion of the Rumin complex on contact manifolds, arXiv:0704.1982.

[10] Troyanov M., Parabolicity of manifolds, Siberian Adv. Math. 9 (1999), 125-150.

[11] Vogan D.A. Jr., Wallach N.R., Intertwining operators for real reductive groups, Adv. Math. 82 (1990), 203-243. 